\title{
Fast inversion of logging-while-drilling resistivity measurements acquired in multiple wells
}

\author{
Shaaban A. Bakr*, David Pardo ${ }^{\dagger}$, and Carlos Torres-Verdín $\ddagger$ \\ *Basque Center for Applied Mathematics (BCAM), Bilbao, Spain. \\ Department of Mathematics, Assiut University, Assiut 71516, Egypt. \\ ${ }^{\dagger}$ University of the Basque Country (UPV/EHU), Leioa, Spain \\ Basque Center for Applied Mathematics (BCAM), Bilbao, Spain. \\ IKERBASQUE (Basque Foundation for Sciences), Bilbao, Spain. \\ $\ddagger$ The University of Texas at Austin, Austin TX, USA.
}

(October 8, 2016)

Running head: LWD Fast Inversion

\begin{abstract}
This paper introduces a new method for the fast inversion of borehole resistivity measurements acquired in multiple wells using logging-while-drilling (LWD) instruments. There are two key novel contributions. First, we approximate general three-dimensional (3D) transversely isotropic (TI) formations with a sequence of several "stitched" one-dimensional (1D) planarly layered TI sections. This allows us to approximate the solution of rather complex 3D formations using only $1.5 \mathrm{D}$ simulations. Second, the developed method supports the simultaneous inversion of measurements acquired in different neighboring wells and/or with different logging instruments.
\end{abstract}

Numerical experiments performed with realistic 3D synthetic formations confirm the flexibility of the method and the reliability of inversion products. The method yields rel- 
ative errors below $5 \%$ on the model space, and it enables the interpretation of resistivity measurements acquired in multiple wells (e.g., an exploratory, an offset, and a geosteering well) and with any combination of co-axial and/or tri-axial commercial logging measurements acquired with known antennae configurations. Numerical results also indicate that thinly-bedded resistive formations are very sensitive to the presence of noise on the measurements and/or to possible errors on bed boundary locations, while conductive layers are only weakly sensitive to those effects. 


\section{INTRODUCTION}

We focus our work on the real-time interpretation of LWD resistivity measurements acquired for well geosteering (Zhou (2015); Dupuis and Denichou (2015); Bittar and Aki (2015)). In high-angle/horizontal wells, discrepancies between apparent and actual resistivities are often large due to the presence of "horns" and other abnormal readings associated with complex geometrical features. For those cases, numerical inversion methods are needed for precise quantitative estimation of the subsurface resistivity distribution, enabling the identification and assessment of hydrocarbon-bearing formations.

In well geosteering, interpretation of resistivity measurements has to be performed in real-time. Typically, over $95 \%$ of the inversion time is spent in the forward modeling (Druskin et al. (1999); Newman and Alumbaugh (2002); Davydycheva et al. (2003); Avdeev et al. (2002); Nam et al. (2008, 2010)), either to compute the solution and/or its derivatives with respect to the model parameters. Thus, the use of efficient forward solvers becomes critical in order to invert LWD resistivity measurements in real time. In this context, general 3D simulation methods are prohibitively expensive from the point of view of computational complexity.

In order to substantially diminish the required simulation and inversion time, several numerical approaches based on various dimensionality reduction techniques have been proposed over the last twenty years (see, for example, Merchant et al. (1996), and Anderson (2001)). Some of them are still used today by most oil companies. More recently, Ijasan et al. (2014) introduced a layer-based inversion workflow to estimate layer-by-layer petrophysical properties. Dyatlov et al. (2015) developed a method for simulation of LWD measurements in $2 \mathrm{D}$ formations based on the boundary integral equations and the Fourier transform, 
which reduces the problem to a series of 1D integral equations. He et al. (2015) presented a new analytic method to compute the response of tri-axial tools in layered formations.

Our starting point is the simulation and inversion method developed in Pardo and Torres-Verdín (2015), which decomposes an original 2D formation (Figure 1a) as a sequence of stitched 1D TI models, where both borehole and mandrel effects are assumed to be negligible (see Figure 1b). Subsequently, we select a different 1D model for each logging position, as described in figures $1 \mathrm{c}$ and $1 \mathrm{~d}$. These $1 \mathrm{D}$ models can be rapidly simulated using existing semi-analytical 1.5D solutions, which are described in Kong (1972). More recent derivations of semi-analytical 1.5D solutions can be found in Løseth and Ursin (2007); Zhong et al. (2008), and He et al. (2015). The resulting approach enables the efficient simulation and inversion of LWD resistivity measurements acquired with a co-axial and/or tri-axial directional resistivity commercial tool in a single well under the assumption of a $2 \mathrm{D}$ TI Earth model with fixed bed boundaries. Most numerical decisions (including the selection of the regularization parameter, weights, stopping criteria, and inversion variable) are automatically determined by the method, thereby relaxing the required numerical expertise of the user. The proposed inversion method is implemented as a FORTRAN90 library, and its highly modular structure enables to combine it with other existing simulators and/or modules that may be needed for particular geophysical applications.

[Figure 1 about here.]

In this work, we extend the inversion method proposed in Pardo and Torres-Verdín (2015) to approach more general 3D formations that approximate more accurately realistic subsurface models. In particular, the resulting software enables simulations of models containing various geological faulting structures in the $x z$ or $y z$ planes, and/or some other 
complex geometrical structures.

A second key contribution of our work is the extension of the inversion library developed in Pardo and Torres-Verdín (2015) to the case of multiple wells and/or logging instruments. In this manner, we are able to jointly invert borehole measurements acquired in several wells and/or with different tools. Such a combination of measurements is often available during actual field operations, as it is nowadays customary to drill exploratory and offset vertical wells where co-axial measurements are acquired, followed by one or various geosteering wells where directional tri-axial measurements are recorded. To perform the inversion, we construct a quadratic cost function that incorporates the weighted contributions of all measurements.

A geometrical model of the subsurface is assumed to be pre-determined, either from nuclear logging measurements or from some other possibly available (prior) information. In either case, we assume that the given bed boundary positions are correct, and we restrict the study only to those situations. Nonetheless, we perform a preliminary numerical study about the sensitivity of inverted measurements to small and/or moderate errors on the bed boundary locations.

To illustrate the performance of the proposed method, we consider two synthetic examples. In both of them, we include three wells, namely: (a) an exploratory (pilot) well and (b) an offset vertical well, which are employed as initial reference of the subsurface formation resistivity properties and also to facilitate the ulterior interpretation of measurements acquired with other wells, and (c) a geosteering high-angle (Zhou (2015)) well that is intended to maximize the posterior fluid production from the reservoir. The developed inversion method starts by using information from pilot/offset-well logs where a simple 1D 
model is often sufficient to interpret the measurements. Then, to successfully interpret resistivity measurements acquired in a geosteering high-angle well, a formation model must be constructed to account for the geologic complexity encountered along the well path. In the first synthetic example, we consider a hydrocarbon-bearing formation with inclined depositional layers and a geological fault that shifts the oil-water contact (OWC) location. In the second example, we include two geological faults along the $x z$ and $y z$ planes. In both cases, the use of 3D geological models and measurements acquired at multiple wells is essential for reliable estimation of subsurface properties.

Due to our lack of fast 3D simulators, in this work we generate measurements employing 1.5D numerical simulations. While it is obvious that there are some discrepancies between $3 \mathrm{D}$ and $1.5 \mathrm{D}$ results, we claim that these differences should be local, given that the considered LWD measurements are only sensitive to the geological model in the proximity of the receivers. To numerically illustrate this point, we have compared $2.5 \mathrm{D}$ against $1.5 \mathrm{D}$ simulation results for a subsurface model that includes a geological fault. Accordingly, we show that discrepancies due to presence of the geological fault only arise over a small segment of the well, and they do not propagate over the entire well. Furthermore, we show that our 1.5D-based inversion method accurately estimates the input resistivity values when using synthetic $2.5 \mathrm{D}$ measurements.

The remainder of this paper is organized as follows: First, we describe the numerical simulation method for the forward problem using a semi-analytical solution. Next, we detail the main components of the inversion method. Results from synthetic examples illustrate the salient features of our inversion algorithm, along with its main limitations. Finally, we emphasize the most preeminent conclusions concerning best practices for the real-time interpretation of borehole resistivity measurements. 


\section{FORWARD METHOD}

\section{D Subsurface Models}

When a geological formation is described in terms of one or several surfaces, each surface is approximated with a grid of rectangles in the $x y$-plane, as depicted in Figure 2. This grid does not need to follow a tensor product structure (Figure 2). Such a feature facilitates the user interaction and construction of 3D models. Each rectangular element of the grid is interpreted as a section of the 3D formation, where materials are assumed to be described in terms of a 1D TI model with a given dip and azimuth. Thus, the original 3D model is described as a sequence of stitched 1D TI models, where both borehole and mandrel effects are assumed negligible (see Figure 1b).

[Figure 2 about here.]

The above representation enables the approximation of rather complex 3D geological structures which may include several geological faults in the $x z$ and/or $y z$ planes. When complex geometrical features are not aligned with the Cartesian coordinates, it is also possible to consider a grid of triangles (rather than rectangles), but this slightly increases the implementation complexity.

\subsection{Semi-Analytical Solution}

For each logging position, we first identify the corresponding $1 \mathrm{D}$ model (see figures 1c, and 1d) by performing a simple search algorithm in the given non-conforming grid of rectangular elements. Then, following Kong (1972), we perform a Hankel transform in the horizontal plane, which reduces the original partial differential wave equation into a 
Helmholtz-type ordinary differential equation (ODE) for each Hankel mode. The resulting ODEs can be easily solved analytically by expressing the solution over each layer as a linear combination of the two existing fundamental solutions, and finding their coefficients by imposing the continuity of (a) the solution and (b) its flux across different material interfaces. The resulting coefficients enforcing proper interface conditions among neighboring layers can be interpreted as transmission and reflection coefficients; recursive formulas exist to compute them efficiently (see, e.g., Kong (1972)). The 3D magnetic field is computed by numerically evaluating the inverse Hankel transform of the above spectral 1D analytical solution by invoking an efficient integration scheme (in our case, we follow Anderson $(1982))$.

To further increase the speed of computations, we group logging positions within a given section of the model according to their azimuth and dip. Those positions exhibiting the same or very similar dip and azimuth (typically, below 0.1 degrees discrepancy) are classified within the same group. Then, for each group, we store most of the information needed to compute the transmission and reflection coefficients corresponding to its first logging position for all Hankel modes. These data can be re-utilized when estimating the magnetic fields associated with the remaining logging positions included within the same group.

\section{Raw Data vs. Postprocessed Data}

With conventional LWD instruments, some components of the magnetic field $\mathbf{H}$ are further post-processed to obtain the so-called "apparent resistivities". The main ideas used to perform this operation are delineated in the works of Bonner et al. (1995) and Clark et al. 
(1988). First, we compute at two different receivers $R X_{1}$ and $R X_{2}$ the z-component of the magnetic field excited by a z-oriented dipole. We denote these quantities as $H_{z z}^{R X_{1}}$ and $H_{z z}^{R X_{2}}$, respectively. $R X_{1}$ is always the receiver closest to the transmitter. Then, we have:

$$
\log \frac{H_{z z}^{R X_{1}}}{H_{z z}^{R X_{2}}}=\underbrace{\log \left(\frac{\left|H_{z z}^{R X_{1}}\right|}{\left|H_{z z}^{R X_{2}}\right|}\right)}_{\text {Attenuation }}+i \underbrace{\left\{p h\left(H_{z z}^{R X_{1}}\right)-p h\left(H_{z z}^{R X_{2}}\right)\right\}}_{\text {Phase difference }},
$$

where $p h$ designates the phase of a complex number.

[Figure 3 about here.]

Finally, for the case of a homogeneous formation, we can compute numerically the relation between the above numbers (attenuation and phase difference) with the formation resistivity, as described in Figure 3. The result of applying this transformation to non-homogeneous formations is called "apparent resistivity".

The developed inversion method supports measurements given either in terms of attenuation and phase differences, or apparent resistivities. The use of the former types of measurements provides the best inversion results in high-angle wells because the transformation from raw data to apparent resistivities is not always surjective, leading to the presence of "horns" on the log. For certain directional components, this transformation is neither injective nor surjective. In these cases, we simply use raw data expressed in terms of attenuation and phase differences. Additionally, the proposed method can invert measurements acquired in one or multiple wells, with co-axial and/or tri-axial directional resistivity instruments. This may include any number of "invalid" measurements within the logs due to the presence of noise and/or other causes that have prevented acquisition of reliable measurements at those logging positions. When selected by the user, the inversion can be restricted to a particular transmitter-receiver combination and/or frequency of operation. 


\section{INVERSION METHOD}

The inversion method requires as input one or various apparent resistivity logs, a description of the logging instrument, the wells trajectories, the measurements acquired per logging position, and a description of the 3D geological model in terms of sections and bed boundaries. It delivers: (a) a layer-by-layer resistivity distribution that minimizes the misfit between the measurements and their numerical simulations, and (b) the corresponding uncertainty bars (also called trust regions) expressing the linearized degree of uncertainty of inversion results.

The main objective of the inverse method is to minimize a quadratic cost functional composed of data misfit and regularization additive terms. For that purpose, we employ a Gauss-Newton method where derivatives are computed using a finite-difference scheme, as described in Pardo and Torres-Verdín (2015). Critical to the performance of this algorithm is the adequate construction of the cost functional. In our case:

- The data misfit is expressed in terms of a weighted $l^{2}$-norm of the logs of the resistivities (denoted as $l_{w}^{2}$ ), where the weights are inversely proportional to the recorded measurements so that all measurements provide a comparable contribution to the cost functional.

- The regularization (stabilization) term is based on the results of the previous iteration. While this decision significantly complicates the mathematical analysis of the method (because the cost functional is not fixed throughout the iterations), it has been shown in a plethora of works to provide adequate results in multiple geophysical problems (see, e.g., Pardo and Torres-Verdín (2015)). In our case, the regularization term

consists of a weighted $L^{2}$-norm (denoted as $L_{w}^{2}$ ) of the difference between the current 
model and that obtained in the previous iteration, thereby preventing large variations in the model from iteration to iteration.

- The regularization (stabilization) parameter used to adjust the relative contribution of the regularization term is also iteration dependent. It is computed based on the analysis of a singular value decomposition of the system of normal equations and the so-called L-curve method (see Hansen (1999)) .

- The measurements input to the inversion are also iteration dependent. In our case, we start with those measurements acquired in neighboring vertical wells. A fast inversion of those measurements provides initial values of resistivities for our $3 \mathrm{D}$ model. Then, we progressively include additional geosteering measurements as data to further guide the inversion. Similarly, the number of layers that form part of the minimization procedure is also iteration dependent. At each iteration, target layers are selected based on the available measurements, thereby avoiding inversion of resistivity values corresponding to layers which are located far away from any available measurement. The user prescribes which measurements and unknowns are considered at each iteration of the inversion procedure.

In mathematical terms, the cost functional corresponding to a fixed set of measurements $m$, a set of simulated measurements $h(\sigma)$, regularization parameter $\lambda$ and regularization prior $\sigma_{0}$ can be expressed as:

$$
C_{w}(\sigma)=\|h(\sigma)-m\|_{l_{w}^{2}}^{2}+\lambda\left\|\sigma-\sigma_{0}\right\|_{L_{w}^{2}}^{2},
$$

whose Gauss-Newton iterations can be described as:

$$
\sigma^{(i+1)}=\sigma^{(i)}-\frac{\operatorname{Re}\left(J, h\left(\sigma^{(i)}\right)-m\right)_{l_{w}^{2}}+\lambda\left(I, \sigma^{(i)}-\sigma_{0}\right)_{L_{w}^{2}}}{\operatorname{Re}(J, J)_{l_{w}^{2}}+\lambda(I, I)_{L_{w}^{2}}}
$$


where $J$ is the Jacobian matrix and $I$ is the identity matrix. In our case, we consider the following Gauss-Newton iterations:

$$
\sigma^{(i+1)}=\sigma^{(i)}-\frac{\operatorname{Re}\left(J, h\left(\sigma^{(i)}\right)-m^{(i)}\right)_{l_{w}^{2}}+\lambda^{(i)}\left(I, \sigma^{(i)}-\sigma^{(i-1)}\right)_{L_{w}^{2}}}{\operatorname{Re}(J, J)_{l_{w}^{2}}+\lambda^{(i)}(I, I)_{L_{w}^{2}}} .
$$

Mathematically, our method consists of a sequence of single Gauss-Newton iterations, where the cost functional corresponding to the $i$-th iteration depends upon the results obtained at the previous iteration. Thus, our cost functional at the i-th iteration is defined as:

$$
C_{w}^{i}(\sigma)=\left\|h(\sigma)-m^{(i)}\right\|_{l_{w}^{2}}^{2}+\lambda^{(i)}\left\|\sigma-\sigma^{(i-1)}\right\|_{L_{w}^{2}}^{2}
$$

\section{NUMERICAL RESULTS}

In this section, we first validate and verify our inversion library via a $2.5 \mathrm{D}$ synthetic example. The procedure illustrates how 1.5D inversion based methods can be employed to invert higher-dimensional LWD measurements. Next, we introduce two realistic synthetic 3D models corresponding to different geological formations and include the presence of geological faults. These models are used to describe the main features and limitations of our proposed $1.5 \mathrm{D}$ inversion library. We also analyze the sensitivity of results with respect to anisotropy and bed-boundary perturbations.

\section{Validation and Verification}

Figure 4 describes an 80-degree deviated well crossing 2D subsurface model that includes a geological fault. Simulation results obtained with a $2.5 \mathrm{D}$ finite-element software are compared to those delivered by our proposed "stitched" sequence of 1D models in Figure 5a. Results indicate that our $1.5 \mathrm{D}$ solution method is properly approximating the $2.5 \mathrm{D}$ simulated measurements everywhere except in the proximity of the geological fault, where $2 \mathrm{D}$ 
effects cannot be completely reproduced via 1.5D simulations. Nonetheless, those higherdimensional effects are local, as expected in LWD resistivity measurements. Furthermore, Figure 5b shows that "our stitched" $1.5 \mathrm{D}$ approximation can be successfully employed to invert 2.5D measurements for the example under consideration, where estimated resistivities closely approximate original values.

[Figure 4 about here.]

[Figure 5 about here.]

\section{Model I}

In our first synthetic model, we consider three wells, namely, (a) an exploratory (vertical) well, (b) an offset (vertical) well, and (c) a geosteering (high-angle) well. Figure 6 includes three different views of our synthetic 3D model formation along with the three well trajectories. Figure 6a focuses on the exploratory well; the offset well is described in Figure 6b, while the geosteering well trajectory is displayed in Figure 6c. In the geosteering well, the dip angle is between 82 and 91 degrees with respect to the vertical direction. In this example, the two vertical wells are used to obtain an initial estimate of layer resistivities, while the geosteering well is invoked to extrapolate such initial model to the areas surrounding the geosteering well trajectory. The geological model includes layers of various thicknesses and resistivity contrasts. The thicknesses of the thinnest and thickest layers are $0.38 \mathrm{~m}$, and $1.52 \mathrm{~m}$, respectively, while the formation resistivity ranges from $0.5 \Omega \cdot \mathrm{m}$ to $150 \Omega \cdot \mathrm{m}$. This synthetic example is intended to appraise the effectiveness of the inversion algorithm with a realistic geological formation and multiple wells. 
[Figure 6 about here.]

Figure 7 displays the corresponding inversion results. Specifically, Figure 7a describes the results obtained from the inversion of only the measurements acquired in the exploratory well (Figure 6a). In this case, we observe an accurate estimation of actual resistivities corresponding to the layers that are navigated by the exploratory well. We subsequently incorporate into the inversion measurements corresponding to the offset well, leading to the results shown in Figure 7b. Next, we consider all the available measurements (including those acquired along the geosteering well) to perform the final inversion. The corresponding results are described in Figure 7c. Inverted resistivities match the actual ones. While this example contains two vertical and one high-angle well, the inversion algorithm can be executed with any number of vertical (even zero) and/or high-angle wells. From the mathematical point of view, the inclusion of additional measurements into the inversion process is achieved by simply incorporating new data points with their own weights into the iteration-dependent cost functional.

[Figure 7 about here.]

When adding a 5\% Gaussian noise to the measurements, the corresponding trust regions (often expressed in terms of error bars) described in Figure 8 indicate that measurements acquired across thinly bedded resistive layers are very sensitive to measurement noise in comparison to those acquired across conductive layers, whose inverted resistivities are almost insensitive to the aforementioned noise level.

[Figure 8 about here.] 
In the above numerical experiments, we have assumed that bed boundary locations correspond exactly to those employed when simulating the measurements. However, estimated bed boundary locations may be inaccurate. In what follows, we quantify the sensitivity of inverted results to different errors on bed boundary locations. Specifically, Figure 9 describes the inverted resistivities when all bed boundary locations are misplaced by simultaneously shifting all of them in one direction by $0.0254 \mathrm{~m}$ (1 in.), $0.0508 \mathrm{~m}$ (2 in.), and $0.116 \mathrm{~m}$ (4 in.), respectively. We observe a progressive deterioration of inverted results as errors on bed-boundary locations increase, especially across thinly bedded resistive layers. Nonetheless, inverted results across most layers are still good approximations of actual resistivity values. Figure 9 shows that several apparently thinly bedded layers exhibit relatively small error bars. However, they may actually correspond to thick beds because the thicknesses shown in the figure only describe the logging length of the well trajectory across that particular layer. Thus, uncertainty of these resistivity values may be low due to measurements recorded on other wells and/or along other sections of the well trajectory.

[Figure 9 about here.]

Finally, we analyze the case of transversely isotropic (TI) rocks. Figure 10a describes the inversion results obtained with measurements acquired in the exploratory well. Horizontal resistivities are properly estimated, whereas vertical resistivities cannot be estimated due to the insensitivity of the measurements acquired in a vertical well to anisotropy, as expected. Similar results are observed along the offset well (see Figure 10b). However, when measurements acquired in the geosteering well (Figure 10c) are incorporated into the inversion, the corresponding results properly estimate both horizontal and vertical resistivities.

[Figure 10 about here.] 


\section{Model II}

In our second synthetic model, we again consider an exploratory (vertical) well, an offset (vertical) well, and a geosteering (high-angle) well. Figure 11 describes the model formation with several geological faults. In all wells, we assume a commercial LWD instrument equipped with two transmitters, two receivers, and one frequency of operation. Logging measurements are acquired every half a foot along well trajectories.

[Figure 11 about here.]

As in our previous example, the developed library first inverts the measurements acquired in the exploratory well, followed by those acquired in the offset well and the geosteering well. Figure 12 shows that inverted results coincide with actual (original) values, thereby verifying the robustness and stability of the proposed inversion algorithm.

[Figure 12 about here.]

We now quantify the sensitive of inversion results to possible miss-alignments of bed boundary locations. Figure 13 describes the inverted resistivities when all bed boundary locations are misplaced by $0.0254 \mathrm{~m}$ (1 in.), $0.0508 \mathrm{~m}$ (2 in.), and $0.116 \mathrm{~m}$ (4 in.), respectively. As in the previous example, the greatest errors are observed across thinly bedded resistive layers when bed boundary locations are significantly misplaced.

[Figure 13 about here.]

For the case of transversely isotropic (TI) formations, Figure 14 shows the corresponding inverted resistivity values. Again, vertical resistivities can only be estimated from measure- 
ments acquired in the geosteering well because co-axial measurements acquired in vertical wells are insensitive to the vertical resistivity.

[Figure 14 about here.]

\section{CONCLUSIONS}

We advocate the use of inversion methods for the accurate and reliable interpretation of resistivity measurements acquired in highly deviated wells, which otherwise often exhibit abnormal apparent resistivities that may lead to incorrect assessments of subsurface electrical properties. The proposed inversion library employs a semi-automatic method to interpret borehole resistivity measurements, and allows great flexibility in terms of the 3D formation models that can be considered, and number and types of wells and logging instruments that we can be used as input. Moreover, since the inversion library relies on fast semi-analytical 1.5D simulations, it only takes in average one hour to invert an $800 \mathrm{~m}$ well trajectory section over a laptop equipped with a four core CPU operating at $2.3 \mathrm{GHz}$.

The inversion algorithm assumes fixed bed boundary locations and estimates local layerby-layer resistivities using a gradient-based inversion algorithm, starting with a subset of the measurements (typically those acquired in vertical wells) to establish an initial subsurface resistivity model. These measurements are subsequently complemented with those acquired in high-angle wells to estimate the remaining layer-by-layer resistivities as well as vertical resistivities in the case of TI formations. In that way, we obtain a map of the Earths subsurface resistivity on the proximity of all wells that can be extrapolated or combined with other measurements in order to asses the entire reservoir.

We illustrated the main features of the inversion library with two complex 3D syn- 
thetic models that contained several geological faults together with measurements acquired in three different neighboring wells. Numerical results confirmed that resistive layers are more sensitive than conductive layers to moderate misplacements of bed boundary locations and/or noise in the measurements, as expected from the physics of the measurements. The sensitivity of inversion results to larger misplacements of the bed boundary locations will be addressed in a forthcoming publication.

Future work will incorporate into the inversion library the possibility of automatically adjusting bed boundary locations based on tri-axial directional resistivity measurements. For that purpose, we will implement an efficient method for computation of $1 \mathrm{D}$ derivatives with respect to bed boundary locations. We shall also work on complementing $1.5 \mathrm{D}$ sim-

ulations with $2.5 \mathrm{D}$ ones in local areas where high-dimensionality Earth models cannot be accurately simulated via $1.5 \mathrm{D}$ modeling, which is expected to increase the confidence on the inverted results. Additionally, we will further study the proposed inversion method by using field data.

\section{ACKNOWLEDGMENT}

The work reported in this paper was funded by University of Texas at Austin's Research Consortium on Formation Evaluation, jointly sponsored by Anadarko, Aramco, BakerHughes, BG, BHP Billiton, BP, Chevron, COSL, ConocoPhillips, DEA Deutsche Erdoel AG, Det Norske, ENI, ExxonMobil, Halliburton, Hess, Maersk Oil, Paradigm, Petrobras, PTTEP, Repsol, Southwest Energy, Schlumberger, Shell, Statoil, Total, Weatherford, Wintershall and Woodside Petroleum Limited.

The first and second authors were also partially supported by the European Union's 
Horizon 2020 research and innovation programme under the Marie Sklodowska-Curie grant agreement No 644602, the Project of the Spanish Ministry of Economy and Competitiveness with reference MTM2013-40824-P, the BCAM Severo Ochoa accreditation of excellence SEV-2013-0323, and the Basque Government through the BERC 2014-2017 program and the Consolidated Research Group Grant IT649-13 on "Mathematical Modeling, Simulation, and Industrial Applications (M2SI)". Carlos Torres-Verdín thanks the financial support of the Brian James Jennings Memorial Endowed Chair in Petroleum and Geosystems Engineering. 


\section{REFERENCES}

Anderson, B. I., 2001, Modeling and Inversion Methods for the Interpretation of Resistivity Logging Tool Response: PhD thesis, Delft University of Technology.

Anderson, W. L., 1982, Fast hankel transforms using related and lagged convolutions: ACM Transactions on Mathematical Software (TOMS), 8, no. 4, 344-368.

Avdeev, D. B., A. V. Kuvshinov, O. V. Pankratov, and G. A. Newman, 2002, Threedimensional induction logging problems, part 1: An integral equation solution and model comparisons: Geophysics, 67, 413-426.

Bittar, M., and A. Aki, 2015, Advancement and economic benefit of geosteering and wellplacement technology: The Leading Edge, 34, 524-528.

Bonner, S. D., J. Tabanou, P. Wu, J. Seydoux, K. Moriarty, B. Seal, E. Kwok, and M. Kuchenbecker, 1995, New 2-mhz multiarray borehole-compensated resistivity tool developed for mwd in slim holes: Society of petroleum engineers.

Clark, B., M. Luling, J. Jundt, M. Ross, and D. Best, 1988, A dual depth resistivity measurement for fewd: Presented at the SPWLA 29th Annual Logging Symposium,(Jun. 1988).

Davydycheva, S., V. Druskin, and T. Habashy, 2003, An efficient finite-difference scheme for electromagnetic logging in 3D anisotropic inhomogeneous media: Geophysics, 68, no. $5,1525-1536$.

Druskin, V. L., L. A. Knizhnerman, and P. Lee, 1999, New spectral Lanczos decomposition method for induction modeling in arbitrary 3-D geometry: Geophysics, 64, no. 3, 701706.

Dupuis, C., and J.-M. Denichou, 2015, Automatic inversion of deep-directional-resistivity measurements for well placement and reservoir description: The Leading Edge, 34, 504- 
512.

Dyatlov, G., E. Onegova, and Y. Dashevsky, 2015, Efficient 2.5D electromagnetic modeling using boundary integral equations: Geophysics, 80, no. 3, E163-E173.

Hansen, P. C., 1999, The l-curve and its use in the numerical treatment of inverse problems: IMM, Department of Mathematical Modelling, Technical Universityof Denmark.

He, Z., R. C. Liu, K. Huang, and C. Guo, 2015, A fast algorithm to forward model the triaxial induction logging response in layered transversely isotropic dipping formations: Geophysics, 80, no. 3, D237-D245.

Ijasan, O., C. Torres-Verdin, and W. E. Preeg, 2014, Inversion-based petrophysical interpretation of logging-while-drilling nuclear and resistivity measurements: Geophysics, 79, no. 3, D145-D159.

Kong, J., 1972, Electromagnetic fields due to dipole antennas over stratified anisotropic media: Geophysics, 37, no. 6, 985-996.

Løseth, L. O., and B. Ursin, 2007, Electromagnetic fields in planarly layered anisotropic media: Geophysical Journal International, 170, no. 1, 44-80.

Merchant, G., R. Strickland, and C. Jackson, 1996, Enhanced resolution lwd resistivity logs using a new inversion technique: SPWLA 37th Annual Logging Symposium, 16-19.

Nam, M. J., D. Pardo, and C. Torres-Verdín, 2008, Simulation of Dual-Laterolog Measurements in Dipping, Invaded and Anisotropic Formations Using a Fourier Series Expansion in a Non-Orthogonal System of Coordinates and a Self-Adaptive $h p$-Finite Element Method: Geophysics., 74, no. 1, E31-E43.

— 2010 , Assessment of delaware and groningen effects on dual-laterolog measurements with a self-adaptive hp finite-element method: Geophysics, 75, no. 6, F143-F149.

Newman, G. A., and D. L. Alumbaugh, 2002, Three-dimensional induction logging prob- 
lems, part 2: A finite-difference solution: Geophysics, 67, no. 2, 484-491.

Pardo, D., and C. Torres-Verdín, 2015, Fast 1D inversion of logging-while-drilling resistivity measurements for improved estimation of formation resistivity in high-angle and horizontal wells: Geophysics, 80, E111-E124.

Zhong, L., J. Li, A. Bhardwaj, L. C. Shen, and R. C. Liu, 2008, Computation of triaxial induction logging tools in layered anisotropic dipping formations: Geoscience and Remote Sensing, IEEE Transactions on, 46, no. 4, 1148-1163.

Zhou, J., 2015, Uncertainty in geosteering and interpretation of horizontal wells - the necessity for constraints and geometric models: The Leading Edge, 34, 492-499. 


\section{LIST OF FIGURES}

1 Sketch of: (a) an original 2D geometrical/geological model, (b) a simplified piecewise 1D model used for inversion, and the (c) first and (d) second 1D geological models in the sequence of "stitched" 1D models associated with different logging positions (represented with a black point). . . . . . . . .

2 A non-uniform Cartesian grid of rectangles for a given surface. . . . . . . .

3 Relationship between the resistivity of the formation and the attenuation in a homogeneous formation for two frequencies (2 and $0.4 \mathrm{MHz})$ and a particular transmitter-receiver spacing of a LWD resistivity instrument. . . . . . . . .

4 A 2D model formation with a geological fault. The well trajectory with a dip angle of 80 degrees is described with the red dashed line. . . . . . . . .

$5 \quad$ Results corresponding to the borehole condition described in Figure 4. (a) Comparison of numerical results obtained with the sequence of "stitched" $1.5 \mathrm{D}$ models against those obtained with a $2.5 \mathrm{D}$ simulator expressed in terms of attenuation and phase differences. (b) Inversion results delivered by our proposed 1.5D library when simulating input measurements with $2.5 \mathrm{D}$ finiteelement software. Red lines identify actual resistivities, while black dashed lines describe estimated resistivities. . . . . . . . . . . . . .

6 Description of geological Model I and three well trajectories. The model includes an oil-bearing formation (red color) limited by a water-bearing rock (blue). The oil-water contact interface is vertically shifted due to an existing geological fault. (a) View of the subsurface model from the proximity of the exploratory vertical well, (b) view of the subsurface model from the proximity of the offset vertical well, and (c) view of the geological model and the trajectory of the geosteering well. . . . . . . . . . . . . .

$7 \quad$ Model I: Inversion results corresponding to the 3D formation model and well trajectories described in Figure 6. Red lines identify actual resistivities, while black dashed lines correspond to estimated resistivities. (a) Inversion results obtained with measurements acquired in the exploratory well. (b) Inversion results obtained after incorporating the measurements acquired in the offset well. (c) Inversion results obtained after incorporating the measurements acquired in the geosteering well. . . . . . . . . . . . . .

8 Trust region (indicated by the blue lines) of inversion results obtained for measurements acquired in the geosteering well that navigated through the $3 \mathrm{D}$ formation model described in Figure 6. . . . . . . . . . . . .

9 Model I: Inversion results (black dashed lines) obtained from geosteeringwell measurements acquired in the formation described in Figure 6 when bed boundary positions are misplaced by (a) $0.0254 \mathrm{~m}$, (b) $0.0508 \mathrm{~m}$, and (c) $0.116 \mathrm{~m}$, respectively. . . . . . . . . . . . . . . 
10 Model I: Inversion results obtained for the 3D TI formation and well trajectories described in Figure 6. Red lines identify actual resistivities, while black lines correspond to estimated resistivities. Dashed and continuous lines identify vertical and horizontal resistivities, respectively. (a) Inversion results obtained with measurements acquired in the exploratory well. (b) Inversion results obtained after incorporating the measurements acquired in the offset well. (c) Inversion results obtained after incorporating the measurements acquired in the geosteering well. . . . . . . . . . . .

11 Description of geological Model II and three well trajectories. The model describes a continuous oil-bearing formation (red color) that is vertically shifted due to the presence of two geological faults aligned along an $x z$ and a $y z$ plane, respectively. (a) View of the subsurface model along with the three well trajectories, (b) view of the formation in the proximity of the geological fault along the $x z$ plane, and (c) view of the subsurface model in the proximity of the geological fault along the $y z$ plane. . . . . . . . . . .

12 Model II: Inversion results obtained for the 3D formation model and well trajectories described in Figure 11. Red lines identify actual resistivities, while black dashed lines correspond to estimated resistivities. (a) Inversion results obtained from measurements acquired in the exploratory well. (b) Inversion results obtained after incorporating the measurements acquired in the offset well. (c) Inversion results obtained after incorporating the measurements acquired in the geosteering well. . . . . . . . . . . . .

13 Model II: Inversion results (black dashed lines) obtained with measurements acquired in the geosteering well across the formation model described in Figure 11 when bed boundary locations are misplaced by (a) $0.0254 \mathrm{~m}$, (b) $0.0508 \mathrm{~m}$, and (c) $0.116 \mathrm{~m}$, respectively. . . . . . . . . . .

14 Model II: Inversion results obtained for the 3D TI formation model and well trajectories described in Figure 11. Red lines identify actual resistivities, while black lines correspond to estimated resistivities. Dashed and continuous lines identify vertical and horizontal resistivities, respectively. (a) Inversion results obtained with measurements acquired in the exploratory well. (b) Inversion results obtained after incorporating the measurements acquired in the offset well. (c) Inversion results obtained after incorporating the measurements acquired in the geosteering well. . . . . . . . . . . 


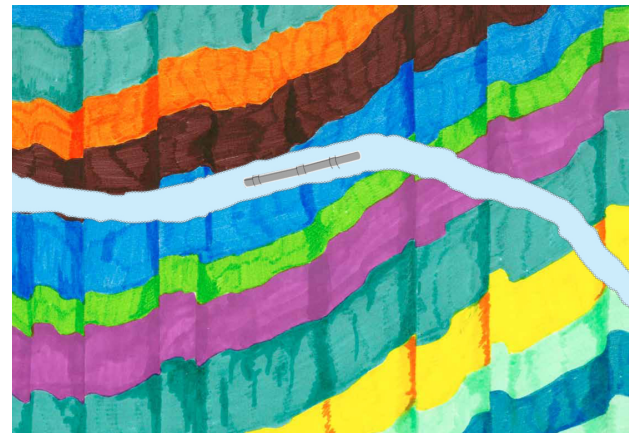

(a) Original 2D model

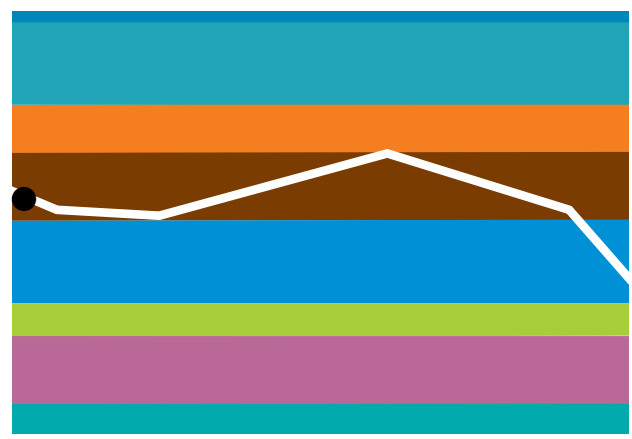

(c) $1 \mathrm{D}$ model for Section 1

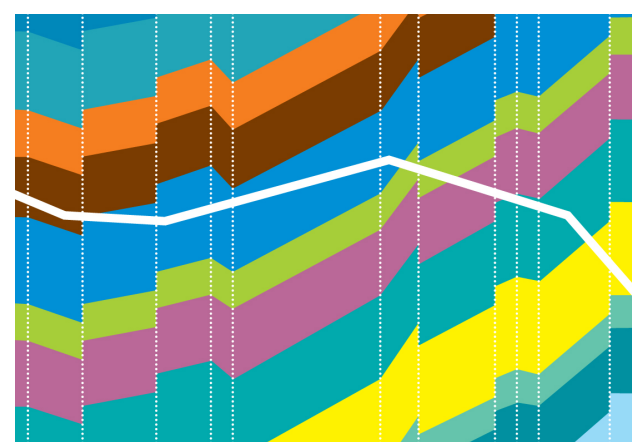

(b) Simplified piecewise 1D model

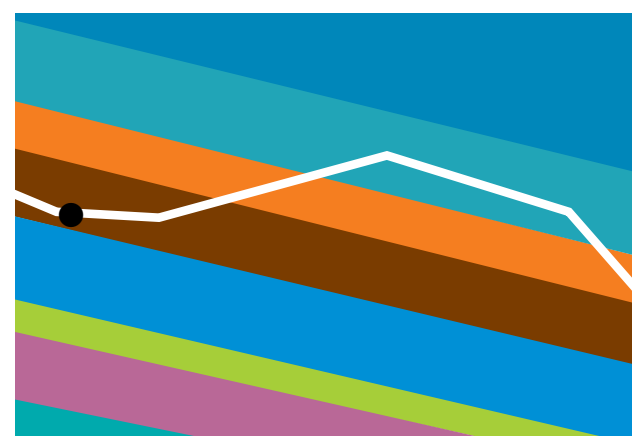

(d) $1 \mathrm{D}$ model for Section 2

Figure 1: Sketch of: (a) an original 2D geometrical/geological model, (b) a simplified piecewise 1D model used for inversion, and the (c) first and (d) second 1D geological models in the sequence of "stitched" 1D models associated with different logging positions (represented with a black point). 


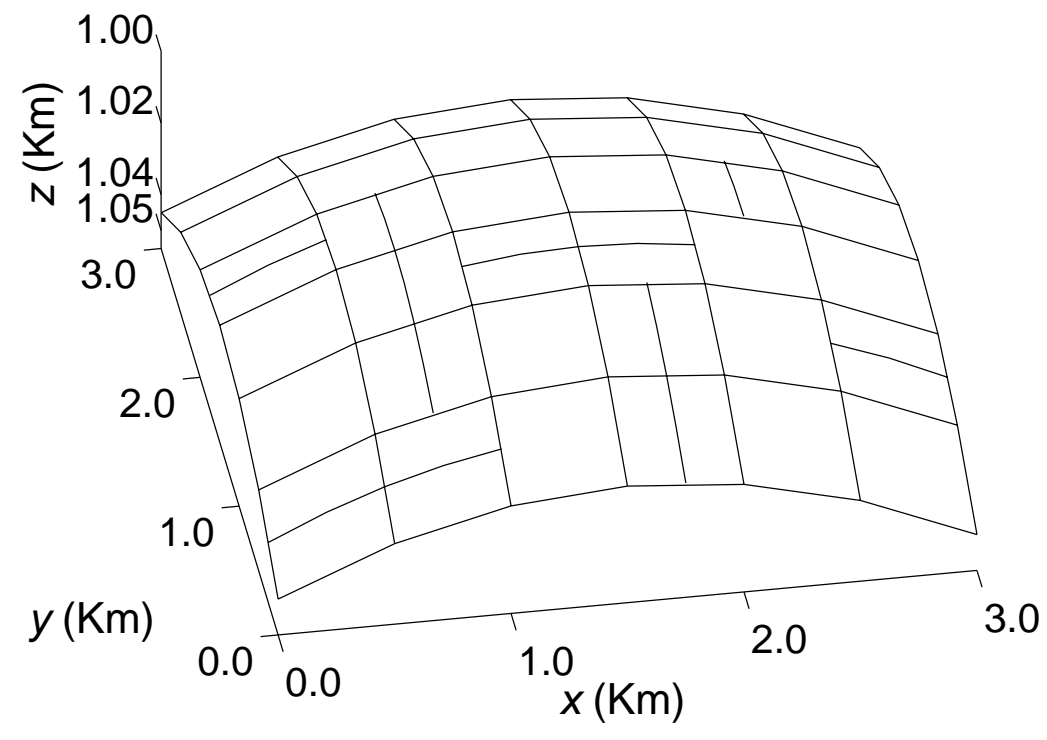

Figure 2: A non-uniform Cartesian grid of rectangles for a given surface. 


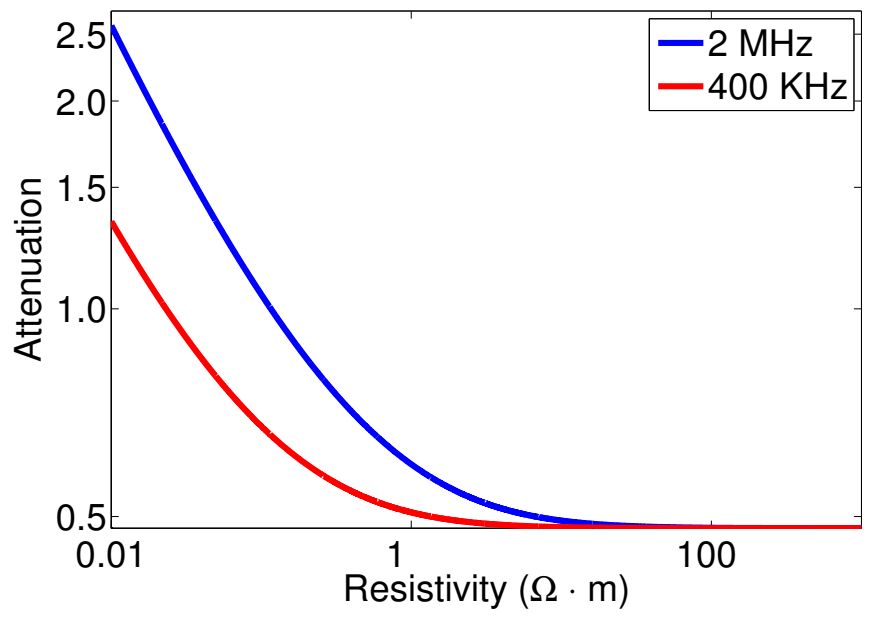

Figure 3: Relationship between the resistivity of the formation and the attenuation in a homogeneous formation for two frequencies (2 and $0.4 \mathrm{MHz})$ and a particular transmitterreceiver spacing of a LWD resistivity instrument. 


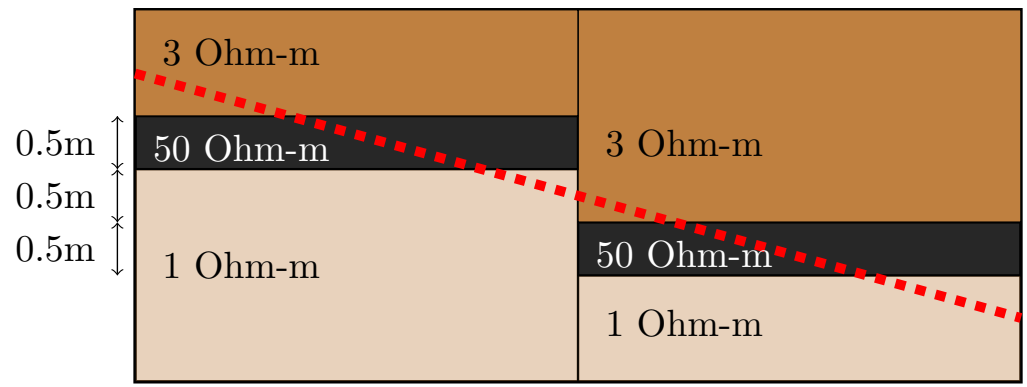

Figure 4: A 2D model formation with a geological fault. The well trajectory with a dip angle of 80 degrees is described with the red dashed line. 


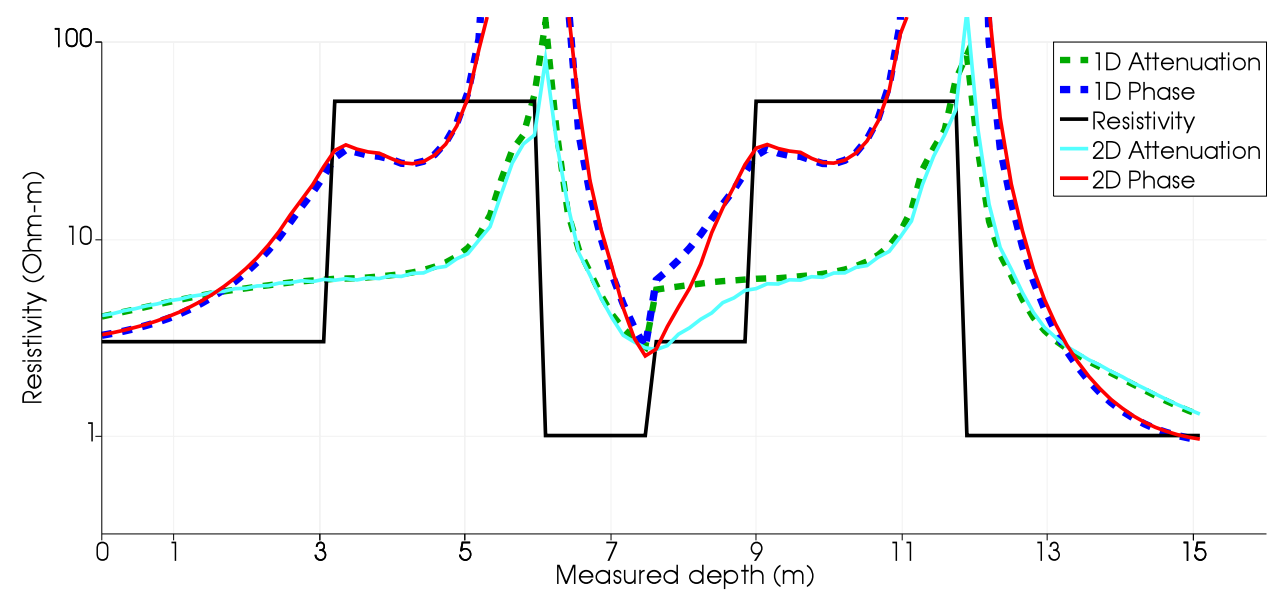

(a) Attenuation and Phase

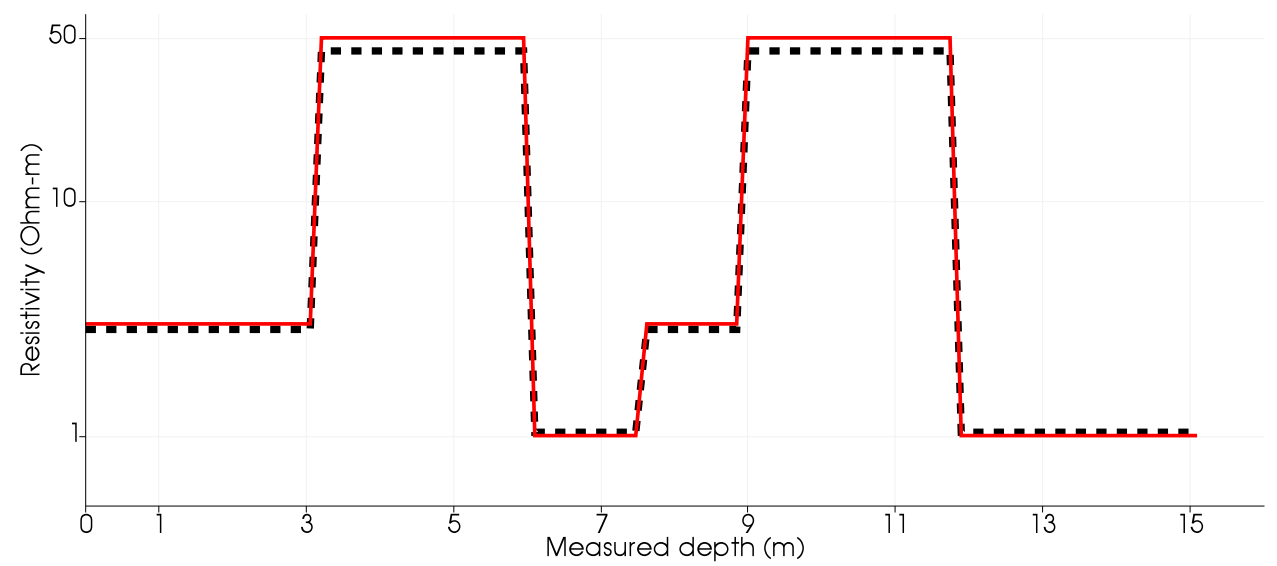

(b) Inversion results

Figure 5: Results corresponding to the borehole condition described in Figure 4. (a) Comparison of numerical results obtained with the sequence of "stitched" 1.5D models against those obtained with a $2.5 \mathrm{D}$ simulator expressed in terms of attenuation and phase differences. (b) Inversion results delivered by our proposed 1.5D library when simulating input measurements with 2.5D finite-element software. Red lines identify actual resistivities, while black dashed lines describe estimated resistivities. 


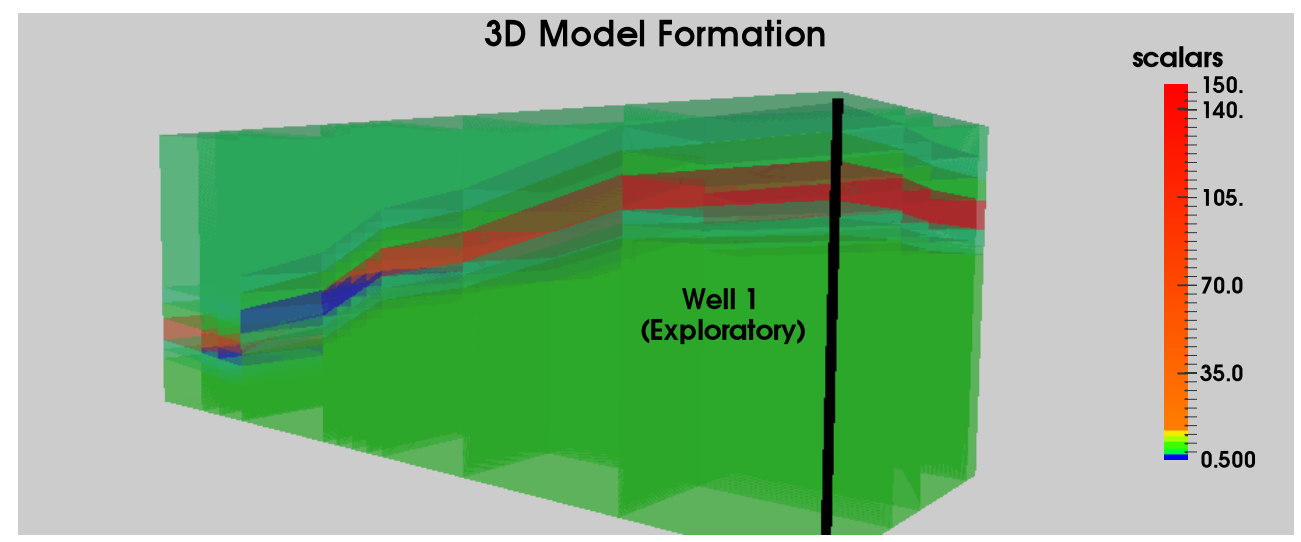

(a) Exploratory Well

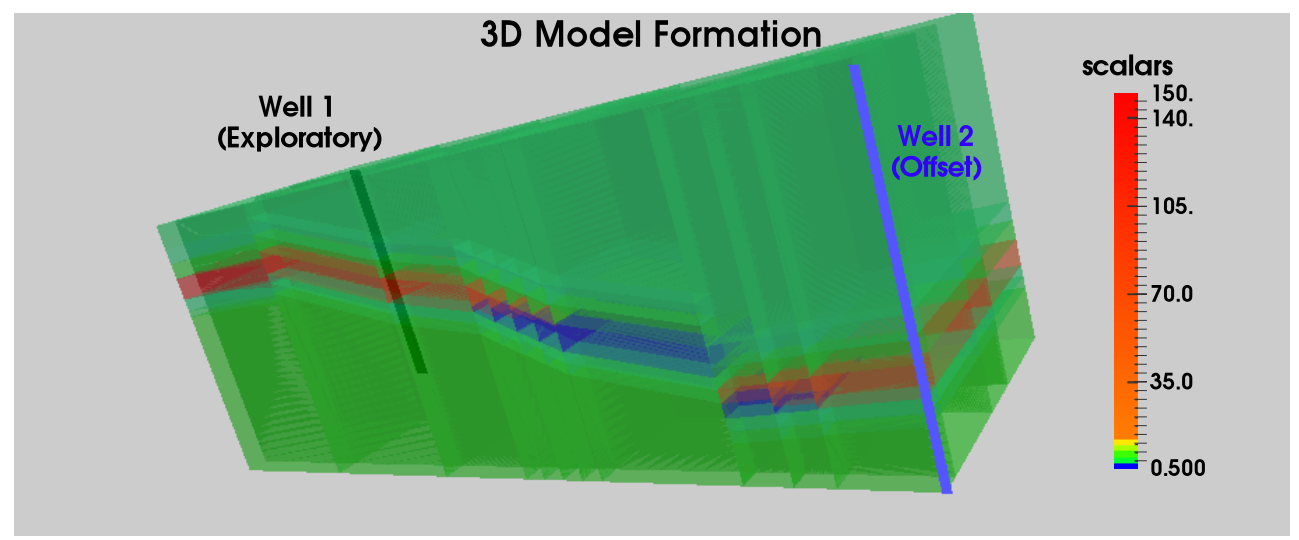

(b) Offset Well

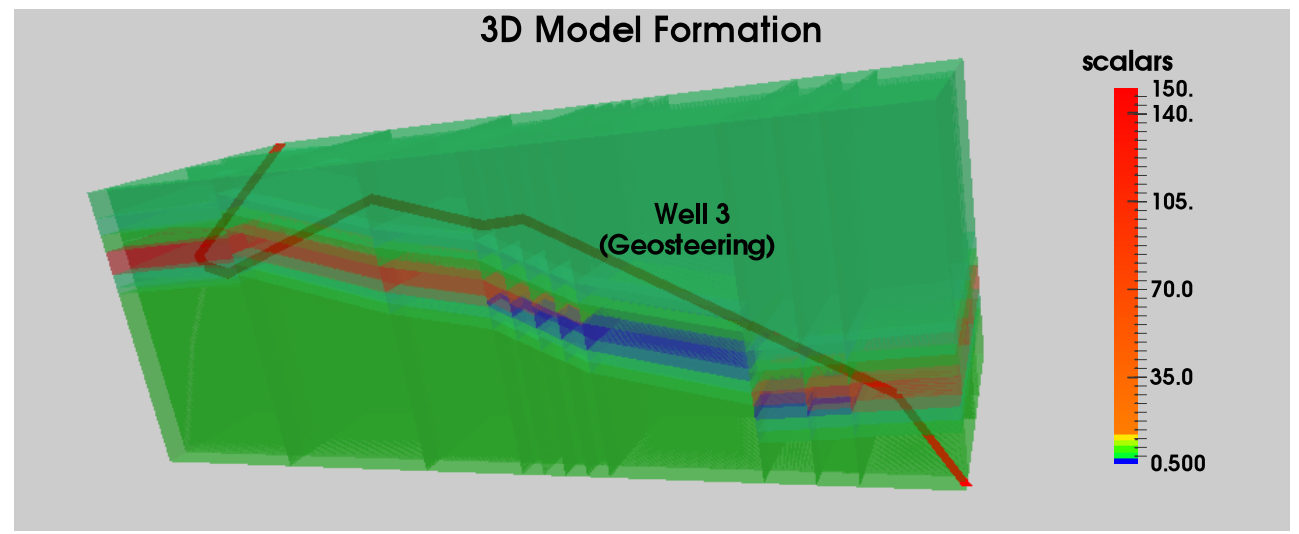

(c) Geosteering Well

Figure 6: Description of geological Model I and three well trajectories. The model includes an oil-bearing formation (red color) limited by a water-bearing rock (blue). The oil-water contact interface is vertically shifted due to an existing geological fault. (a) View of the subsurface model from the proximity of the exploratory vertical well, (b) view of the subsurface model from the proximity of the offset vertical well, and (c) view of the geological model and the trajectory of the geosteering well. 


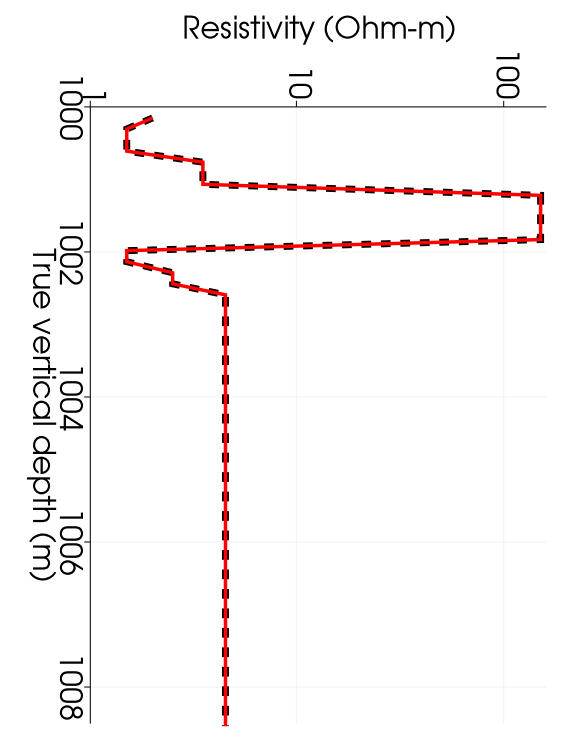

(a) Logs (Exploratory Well)

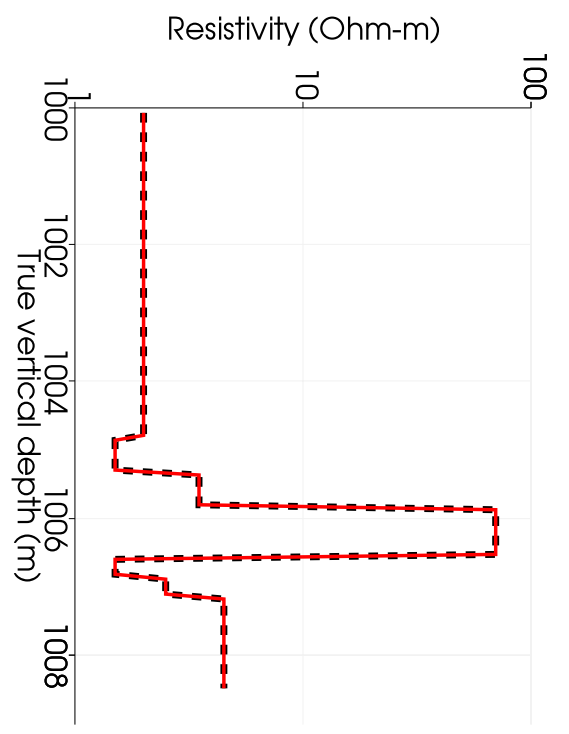

(b) Logs (Offset Well)

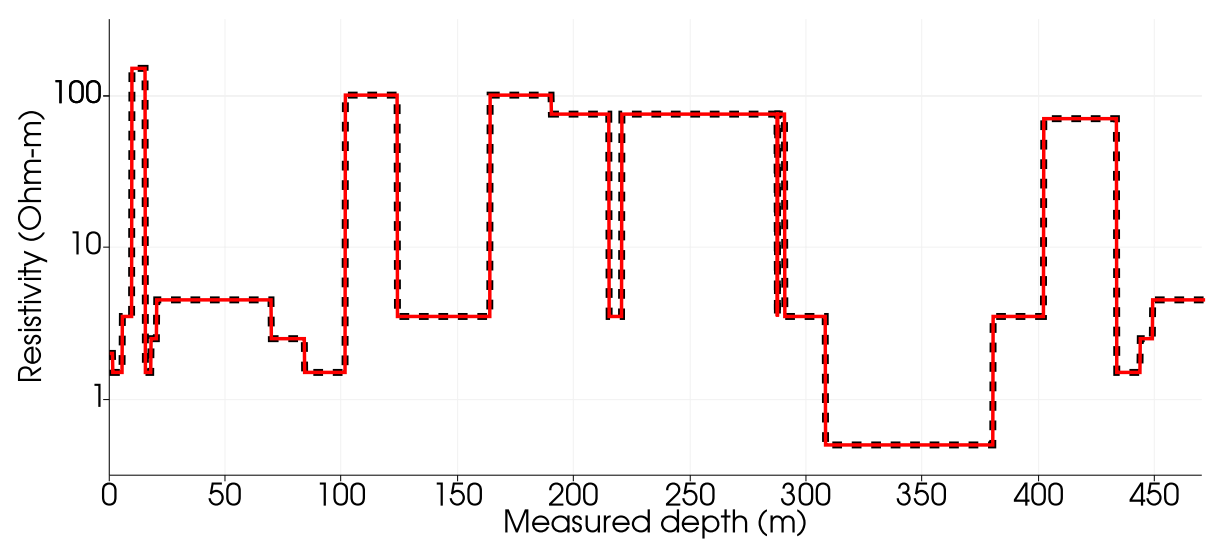

(c) Logs (Geosteering Well)

Figure 7: Model I: Inversion results corresponding to the 3D formation model and well trajectories described in Figure 6. Red lines identify actual resistivities, while black dashed lines correspond to estimated resistivities. (a) Inversion results obtained with measurements acquired in the exploratory well. (b) Inversion results obtained after incorporating the measurements acquired in the offset well. (c) Inversion results obtained after incorporating the measurements acquired in the geosteering well. 


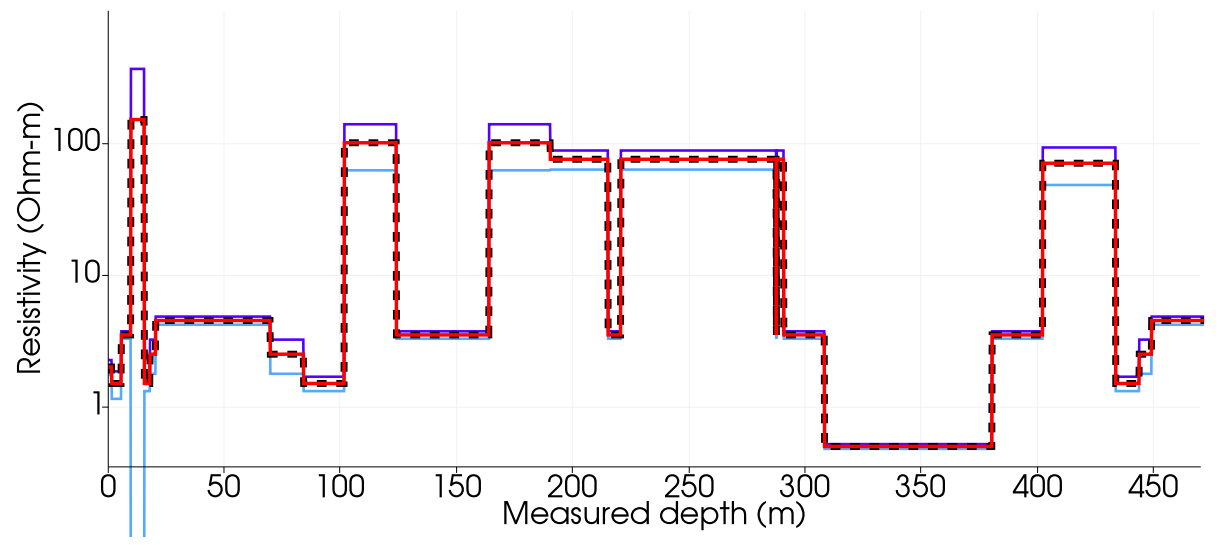

(a) Trust Region (Geosteering Well)

Figure 8: Trust region (indicated by the blue lines) of inversion results obtained for measurements acquired in the geosteering well that navigated through the $3 \mathrm{D}$ formation model described in Figure 6. 


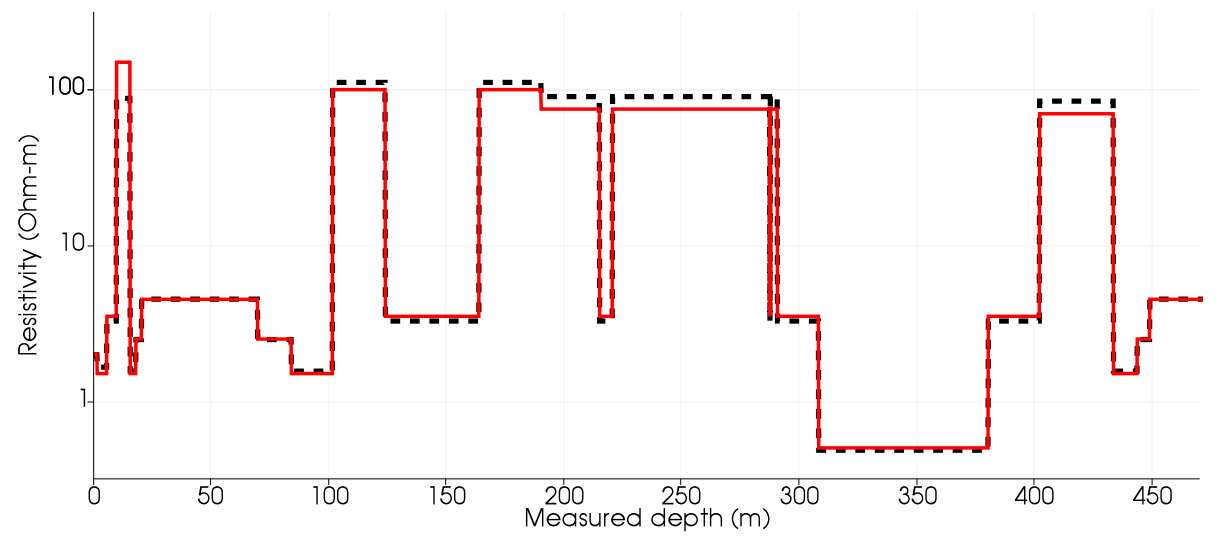

(a) $0.0254 \mathrm{~m}$ (1 inch)

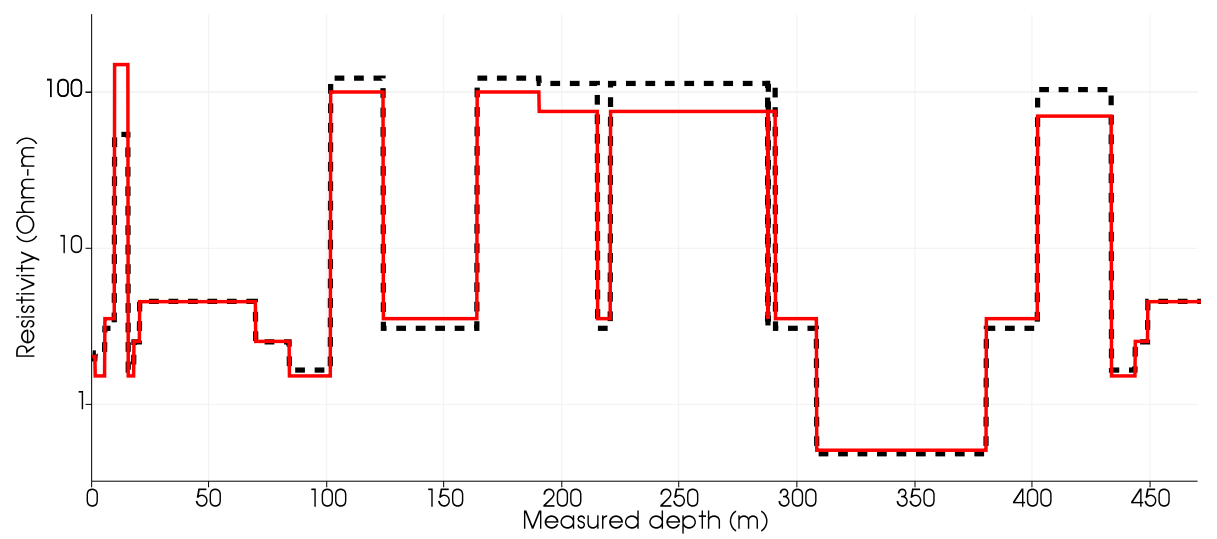

(b) $0.0508 \mathrm{~m}$ (2 inches)

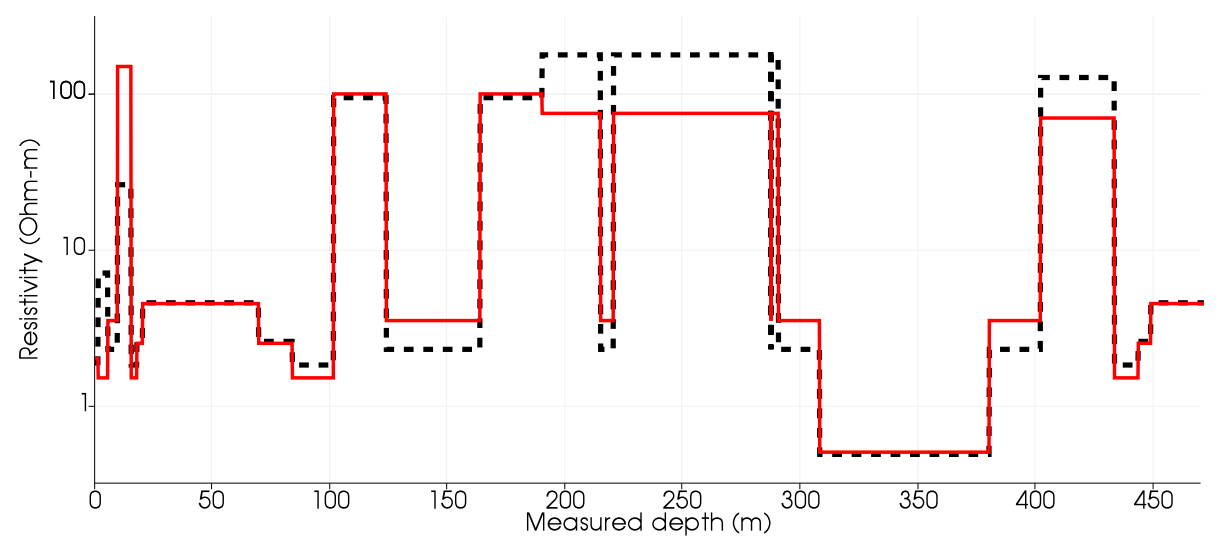

(c) $0.116 \mathrm{~m}$ (4 inches)

Figure 9: Model I: Inversion results (black dashed lines) obtained from geosteering-well measurements acquired in the formation described in Figure 6 when bed boundary positions are misplaced by (a) $0.0254 \mathrm{~m}$, (b) $0.0508 \mathrm{~m}$, and (c) $0.116 \mathrm{~m}$, respectively. 


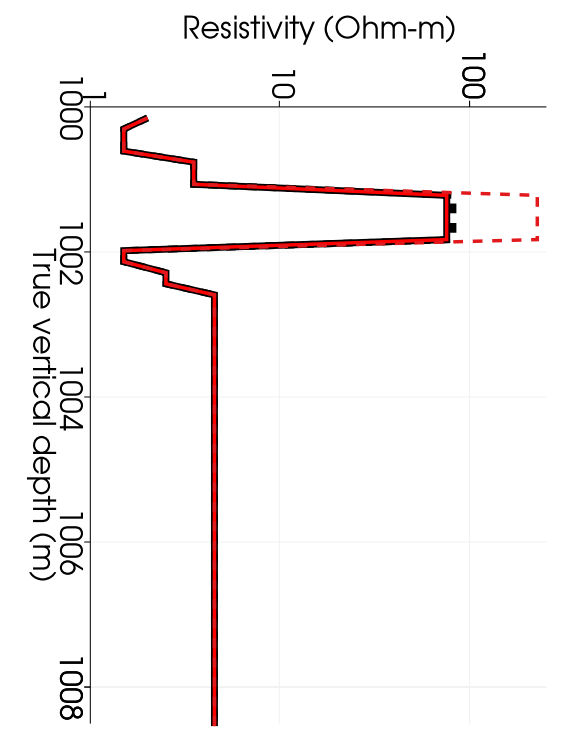

(a) Logs (Exploratory Well)

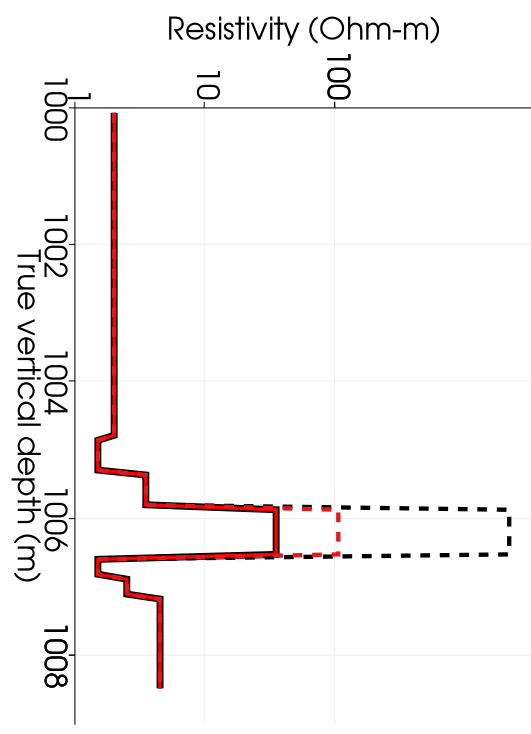

(b) Logs (Offset Well)

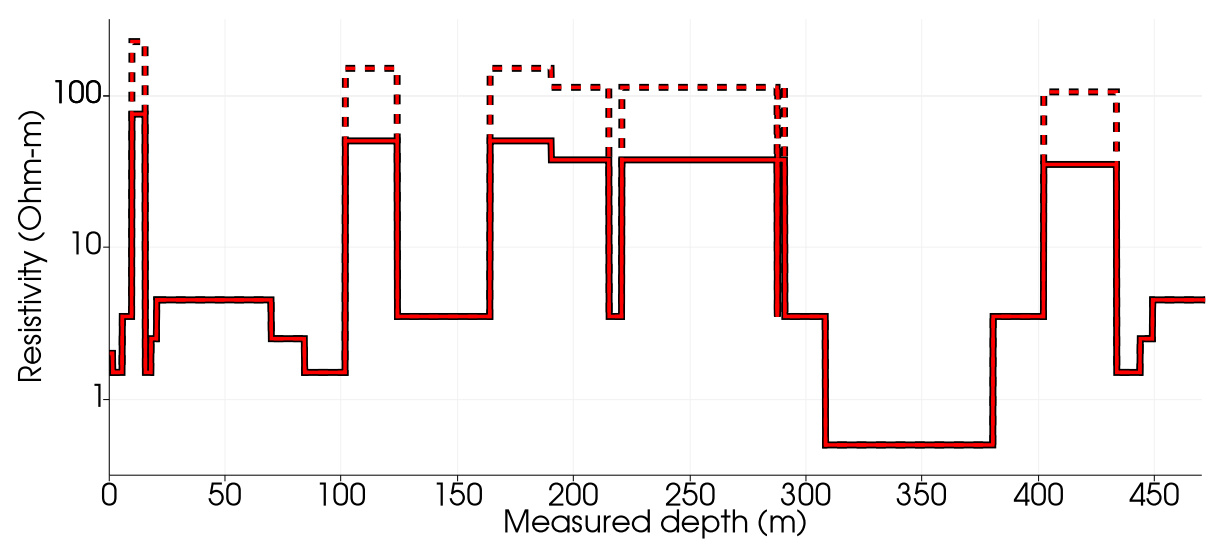

(c) Logs (Geosteering Well)

Figure 10: Model I: Inversion results obtained for the 3D TI formation and well trajectories described in Figure 6. Red lines identify actual resistivities, while black lines correspond to estimated resistivities. Dashed and continuous lines identify vertical and horizontal resistivities, respectively. (a) Inversion results obtained with measurements acquired in the exploratory well. (b) Inversion results obtained after incorporating the measurements acquired in the offset well. (c) Inversion results obtained after incorporating the measurements acquired in the geosteering well. 


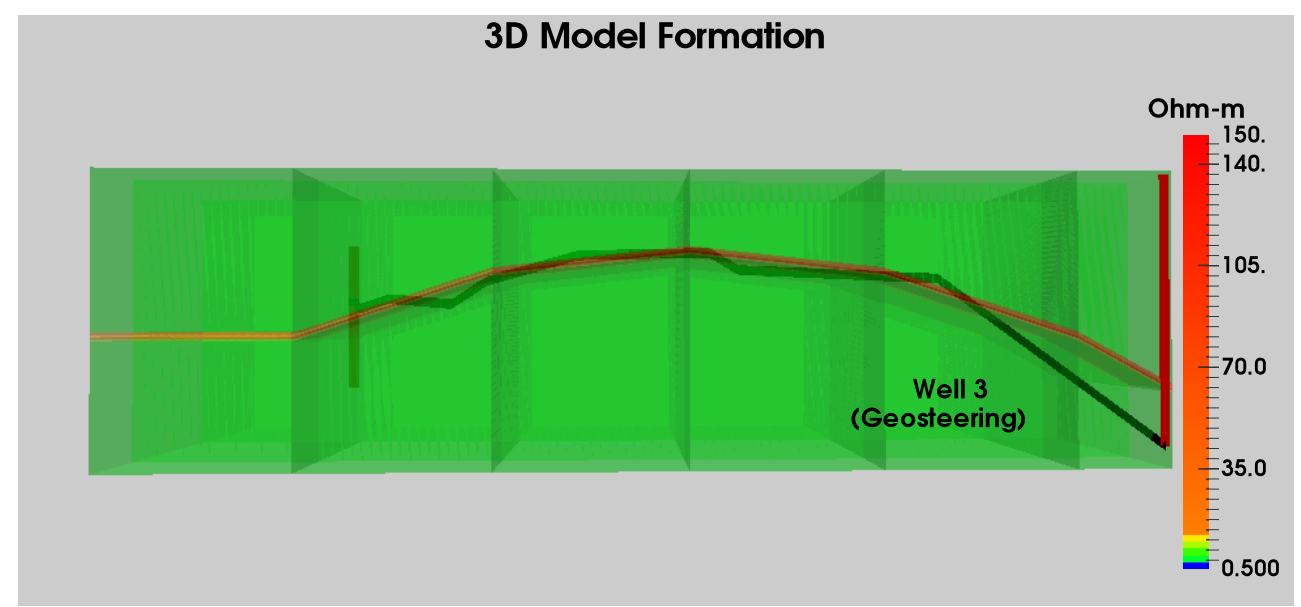

(a) 3D model formation (view 1)

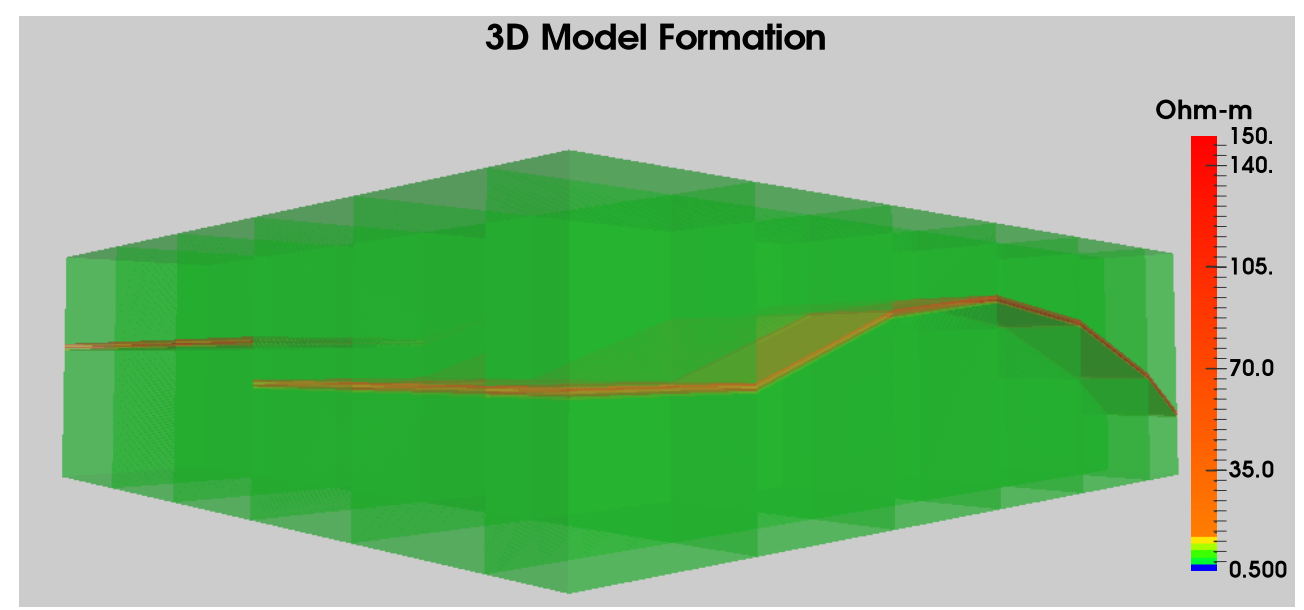

(b) 3D model formation (view 2)

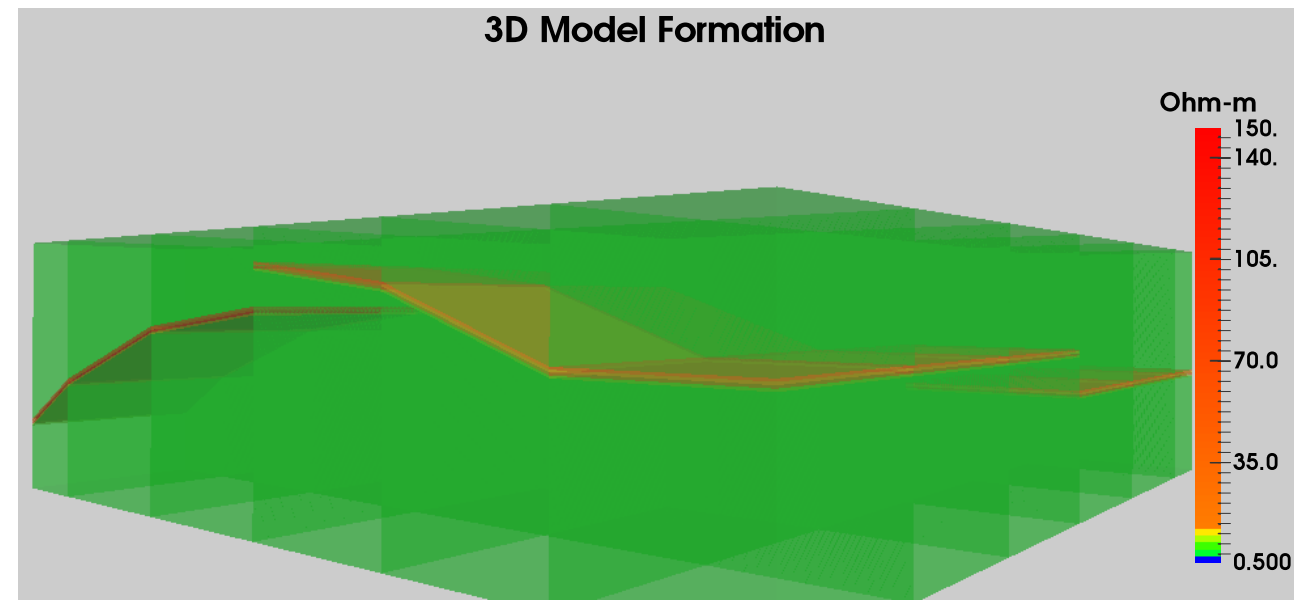

(c) 3D model formation (view 3)

Figure 11: Description of geological Model II and three well trajectories. The model describes a continuous oil-bearing formation (red color) that is vertically shifted due to the presence of two geological faults aligned along an $x z$ and a $y z$ plane, respectively. (a) View of the subsurface model along with the three well trajectories, (b) view of the formation in the proximity of the geological fault along the $x z$ plane, and (c) view of the subsurface model in the proximity of the geological fault along the $y z$ plane. 


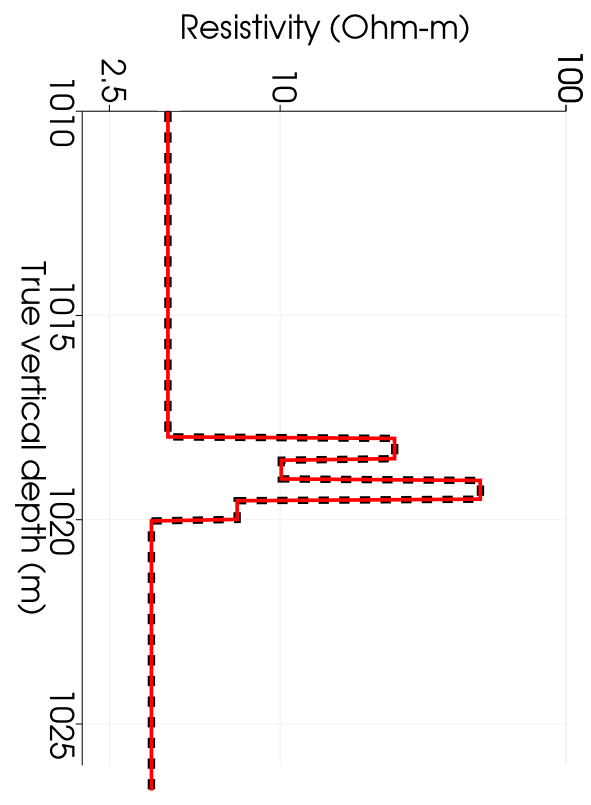

(a) Logs (Exploratory Well)

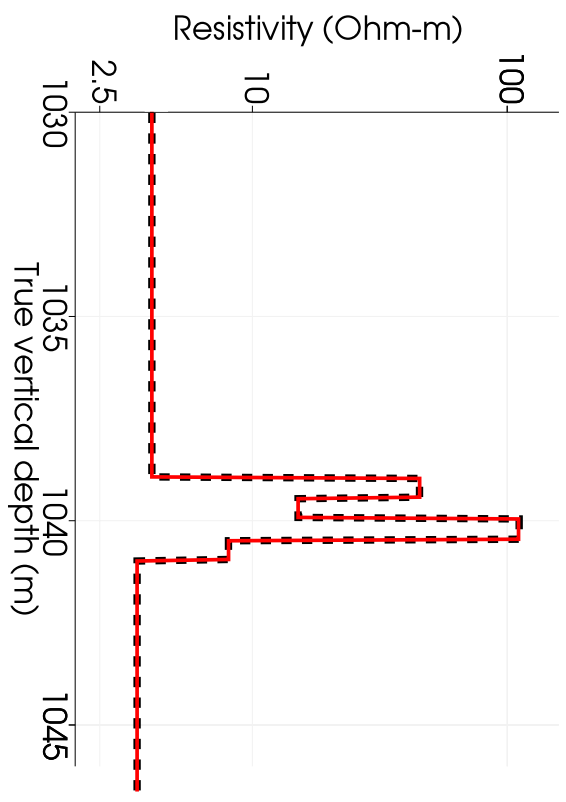

(b) Logs (Offset Well)

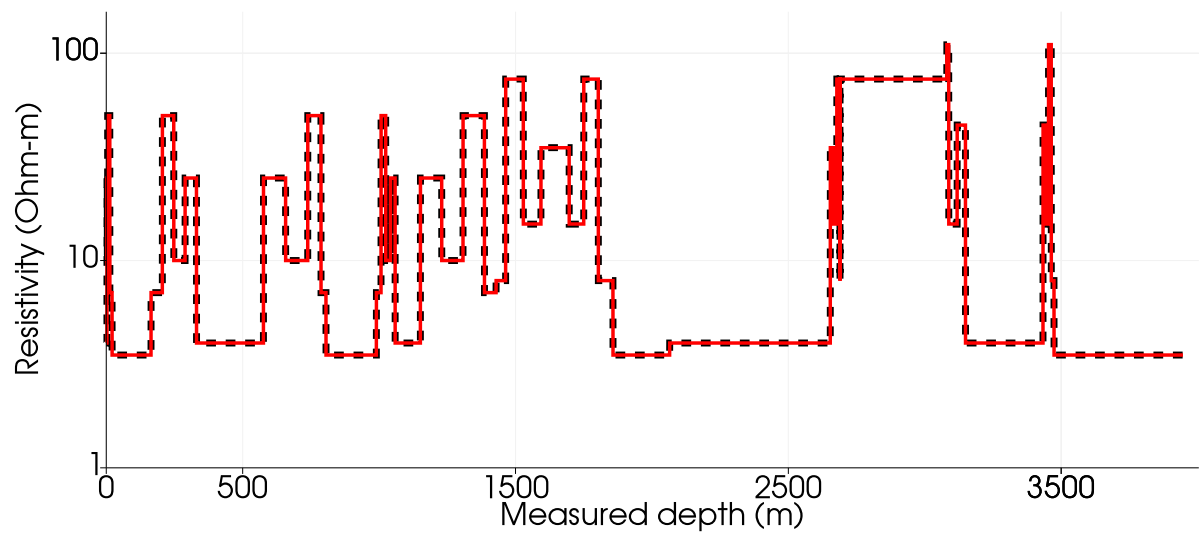

(c) Logs (Geosteering Well)

Figure 12: Model II: Inversion results obtained for the 3D formation model and well trajectories described in Figure 11. Red lines identify actual resistivities, while black dashed lines correspond to estimated resistivities. (a) Inversion results obtained from measurements acquired in the exploratory well. (b) Inversion results obtained after incorporating the measurements acquired in the offset well. (c) Inversion results obtained after incorporating the measurements acquired in the geosteering well. 


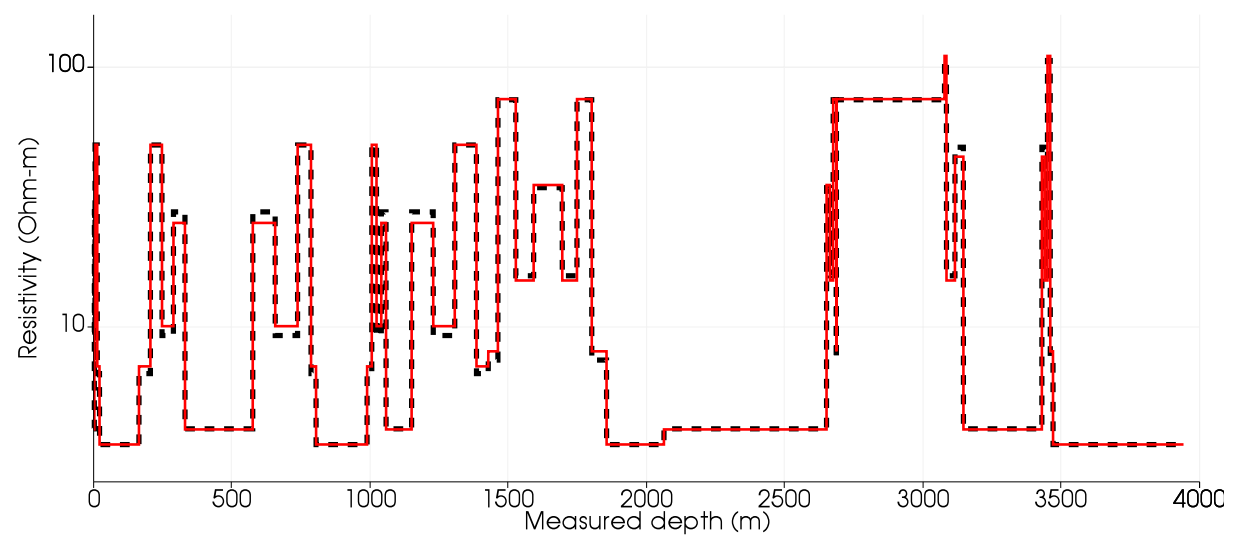

(a) $0.0254 \mathrm{~m}$ (1 inch)

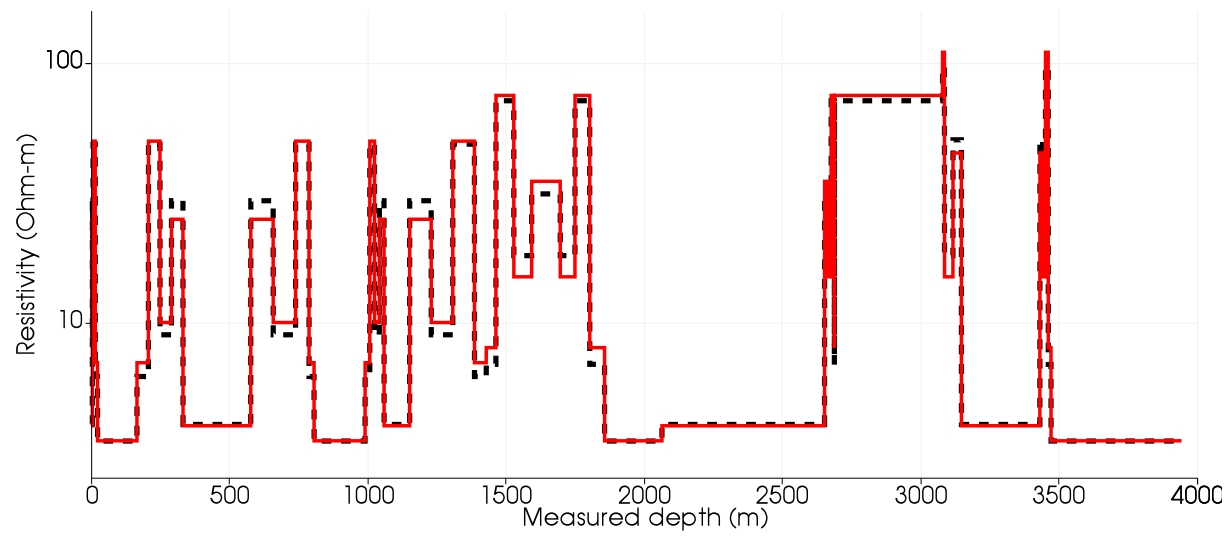

(b) $0.0508 \mathrm{~m}$ (2 inches)

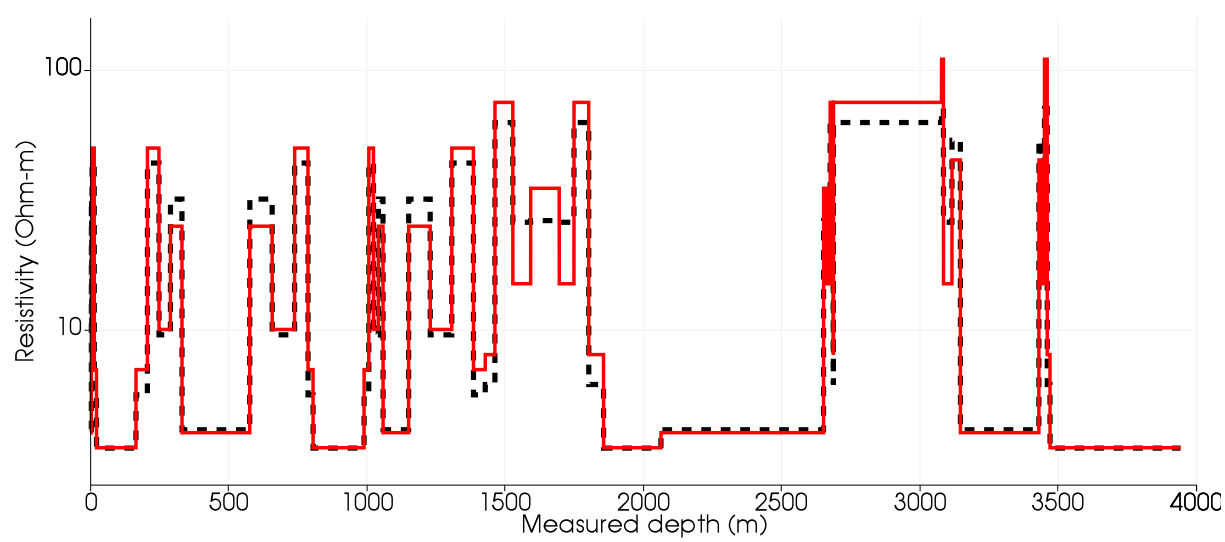

(c) $0.116 \mathrm{~m}$ (4 inches)

Figure 13: Model II: Inversion results (black dashed lines) obtained with measurements acquired in the geosteering well across the formation model described in Figure 11 when bed boundary locations are misplaced by (a) $0.0254 \mathrm{~m}$, (b) $0.0508 \mathrm{~m}$, and (c) $0.116 \mathrm{~m}$, respectively. 


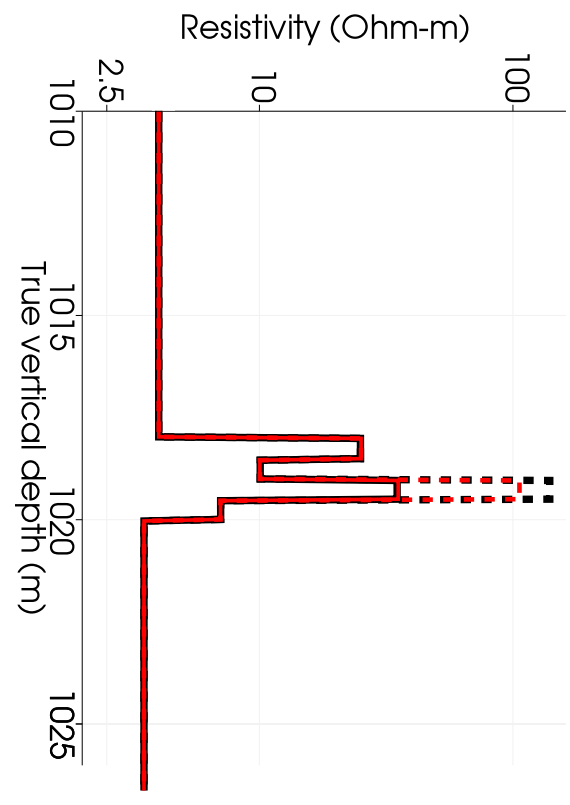

(a) Logs (Exploratory Well)

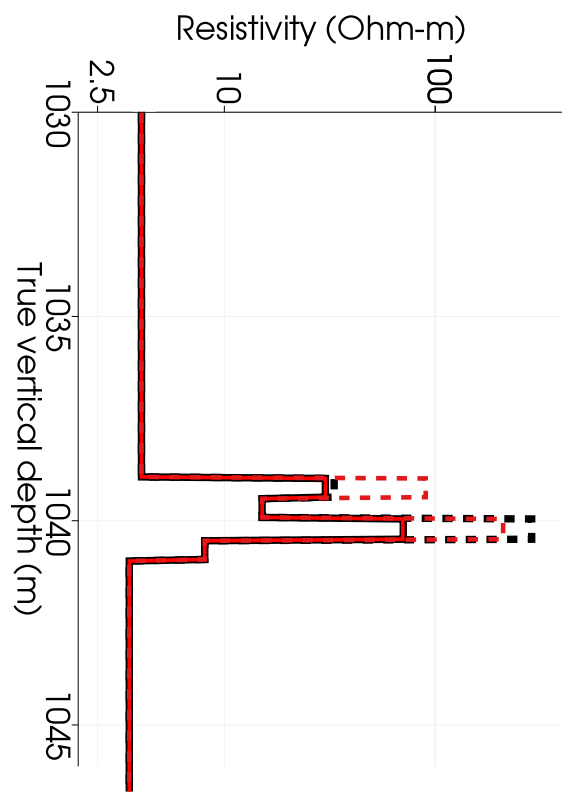

(b) Logs (Offset Well)

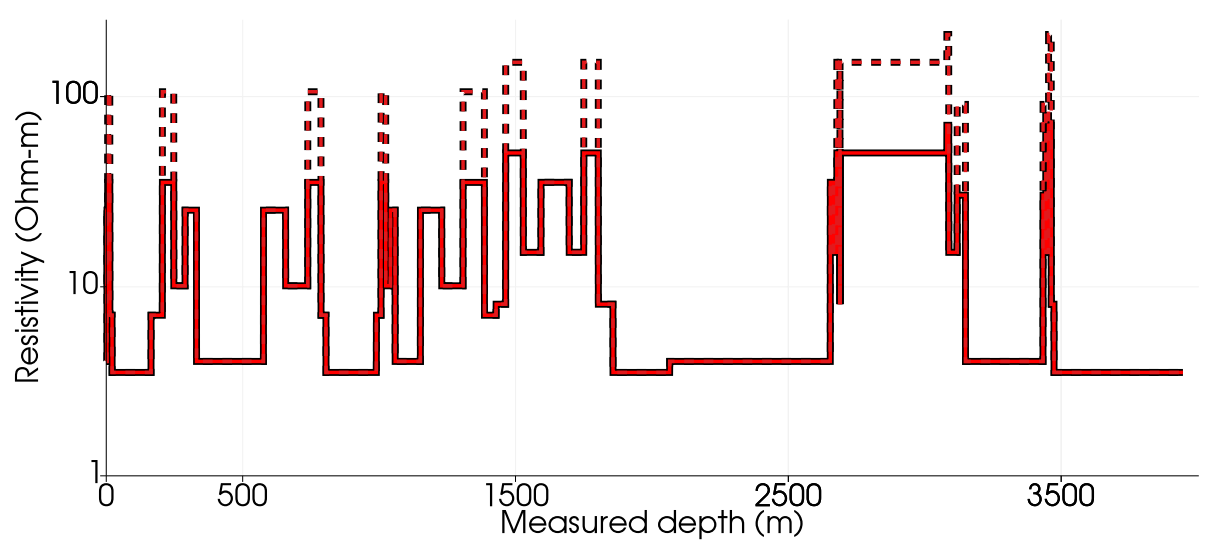

(c) Logs (Geosteering Well)

Figure 14: Model II: Inversion results obtained for the 3D TI formation model and well trajectories described in Figure 11. Red lines identify actual resistivities, while black lines correspond to estimated resistivities. Dashed and continuous lines identify vertical and horizontal resistivities, respectively. (a) Inversion results obtained with measurements acquired in the exploratory well. (b) Inversion results obtained after incorporating the measurements acquired in the offset well. (c) Inversion results obtained after incorporating the measurements acquired in the geosteering well. 